\title{
Microdisk cavities with a Brewster notch
}

\author{
Julius Kullig* and Jan Wiersig $\oplus^{\dagger}$ \\ Institut für Physik, Otto-von-Guericke-Universität Magdeburg, Postfach 4120, D-39016 Magdeburg, Germany
}

(Received 11 March 2021; accepted 18 May 2021; published 11 June 2021)

\begin{abstract}
In a recent paper [J. Kullig, X. Jiang, L. Yang, and J. Wiersig, Phys. Rev. Res. 2, 012072(R) (2020)], the authors reported on an alternative concept to confine light in a microdisk cavity which is based on sequential transmissions at Brewster's angle. In this paper, we combine that approach with the traditional concept of whispering-gallery microcavities which utilize total internal reflection. This allows for a notched cavity design promoting high- $Q$ modes which are conventionally excitable via an attached waveguide and have a considerable fraction of the intensity confined in the notch. The interesting mode characteristics as well as the excellent sensing capability of the Brewster notched cavity is discussed. A further enhancement in the sensitivity is achieved via the utilization of an exceptional point.
\end{abstract}

DOI: 10.1103/PhysRevResearch.3.023202

\section{INTRODUCTION}

Optical microcavities are able to confine light in a very small volume for long times [1,2]. Based on this capability, many interesting applications such as sensors for particles [3-6] or temperature [7-9], gyroscopes [10], microlasers [11-19], frequency comb generators for telecommunication [20-22], and orbital angular momentum lasers [23] have been developed. Additionally, optical microcavities are eminently suited model systems to study wave chaos and non-Hermitian physics [24] which can result in timely innovations like spatiotemporal stable microlasers [25] and broadband waveguide coupling [26].

In the standard approach, the light confinement is achieved via total internal reflection at the cavity's interface. Therefore, in whispering-gallery cavities optical modes with high- and ultrahigh-quality factors are formed [27]. However, in a recent article [28], an alternative concept for the light confinement was introduced: Via sequential transmissions at Brewster's angle, light rays can follow periodic orbits which, alternating, leave and reenter a star-shaped cavity without loss of intensity. Accordingly, in the wave dynamics, long-lived modes are formed which are supported by these periodic light rays. The advantage of the microstar is that a large fraction of the mode's intensity is trapped in the free space where it can interact, e.g., with potential test particles. However, a downside is that the scattering losses at the spikes of the microstar cavity make an in-plane excitation with an attached waveguide rather inefficient.

\footnotetext{
*julius.kullig@ovgu.de

†jan.wiersig@ovgu.de
}

Published by the American Physical Society under the terms of the Creative Commons Attribution 4.0 International license. Further distribution of this work must maintain attribution to the author(s) and the published article's title, journal citation, and DOI.
The aim of this paper is to combine the advantages of the microstar cavity and the in-plane excitability of modes with an attached waveguide as commonly used for whispering-gallery cavities. To do so, a cavity with a properly designed notch is considered where light rays follow periodic orbits which utilize total internal reflection as well as the transmission at Brewster's angle when passing the notch. Thus, in the wave dynamics, not only modes with high-quality factors but also with high intensities in the free space of the notch are achieved, which makes these cavities well suited for particle sensing application.

In addition, we show that the notched cavity can be tuned very close to an exceptional point (EP) [29]. These are points in parameter space of a non-Hermitian system where at least two eigenvalues (frequencies) and simultaneously the corresponding eigenvectors (modes) coalesce. In the recent literature, EPs have been studied intensively due to their interesting physics which manifests, e.g., in copropagating and chiral modes [30-33]. In the parameter space around the EP, the complex frequencies of the involved modes exhibit a characteristic root topology. Therefore, EPs are of particular interest for ultrasensitive sensors [34-37]. In this paper, we achieve an EP by using a fully asymmetric boundary deformation of the notched cavity and verify the expected sensing improvement.

The paper is organized as follows. In Sec. II, we introduce the cavity with a Brewster notch. In Sec. III, the sensing capability of these cavities is discussed. The utilization of an EP to enhance the sensing performance is presented in Sec. IV. A summary is given in Sec. V.

\section{CAVITIES WITH A BREWSTER NOTCH}

\section{A. General setup}

For quasi-two-dimensional microdisk cavities, Maxwell's equations can be formulated as a scalar mode equation [38],

$$
\left[\Delta+k^{2} n^{2}(\vec{r})\right] \psi(\vec{r})=0,
$$



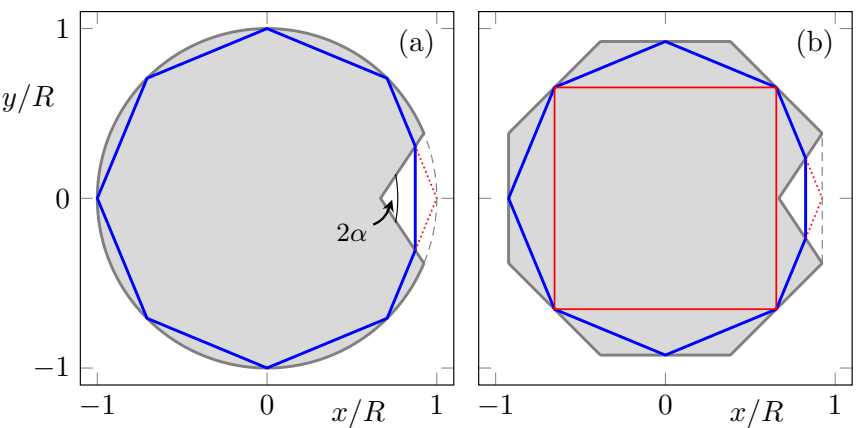

FIG. 1. The gray shaded region indicates the (a) circular-notched and (b) polygonal-notched microdisk. The periodic orbits are shown as colored solid curves. A dotted curve denotes the periodic orbit's reflection at the dashed curve corresponding boundary without notch.

where the wave function $\psi$ either represents the magnetic (transverse electric [TE] polarization) or the electric field (transverse magnetic [TM] polarization). The microdisk is represented by the spacial-dependent effective refractive index $n(\vec{r})$. To utilize Brewster's angle, we here assume TE polarization such that $\vec{H}=\left[0,0, \operatorname{Re}\left(\psi e^{-i \omega t}\right)\right]$ with $\omega=c k ; c$ is the speed of light in vacuum. Thus, at the dielectric interface the wave function $\psi$ as well as the scaled normal derivative $n^{-2} \partial_{\vec{v}} \psi$ need to be continuous. The quality factor of a mode is calculated from the (dimensionless) complex frequency $k R$ by $Q=-\operatorname{Re}(k R) / \operatorname{Im}(2 k R) ; R$ is a typical radius of the cavity.

To design a cavity with a Brewster notch, first, a periodic orbit with $v$ total internal reflections in a whispering-gallery cavity is considered. This might be either a circular cavity or a polygonal cavity. Second, at one of the periodic orbit's reflection points, a notch is introduced to the cavity such that the total internal reflection is replaced by two transmissions as shown in Fig. 1. To remain a periodic orbit, the refractive index of the cavity needs to be adjusted to

$$
n=\frac{1+\sin (\pi / \nu)}{\cos (\pi / \nu)}
$$

and an opening angle of the notch $\alpha=\arctan (n)$ is required. In the shown example with $v=8$, we therefore adjust $n \approx$ 1.4966 and $\alpha=56.25^{\circ}$. This selection also ensures that both transmissions happen at Brewster's angle such that a ray on the periodic orbit does not lose intensity from partial reflections. Note that in the polygonal-notched cavity, additionally a family of period-4 orbits based on total internal reflections exist as shown in Fig. 1(b).

Next, the modes in the notched cavity are analyzed using the boundary element method (BEM) [39]. For the numerical treatment, the sharp corners are smoothed on the angular scale $\delta=0.001$ by a convolution of the cavity's radius function with the kernel $j_{\delta}(\phi) \sim \exp \left[-\delta^{2} /\left(\delta^{2}-\phi^{2}\right)\right] / \delta$. In Fig. 2, the modes of the circular- and polygonal-notched cavities are shown. It is mentioned that the modes occur in pairs of even and odd parities with respect to the symmetry axis of the cavity. For the circular-notched cavity, a variety of long- and short-lived modes exist. The modes with the large $Q$ factor are supported by a transition through the Brewster notch as shown in Figs. 2(c) and 2(d) whereas, in the polygonal-notched cavity, a few long-lived modes are well separated from the rest of the modes with a low $Q$ factor. Thus, there exists a gap between long-lived and short-lived modes as indicated by the shaded region in Fig. 2(b) which is advantageous, e.g., for mode selection [40,41]. The modes with the large $Q$ factor are either supported by a transmission at Brewster's angle [see Fig. 2(f)] or the period-4 orbit [see Fig. 2(e)]. It is worth mentioning that the size of the notch is much larger than the (local) wavelength $\lambda=2 \pi / k$ of the mode. Therefore, the mode discrimination here is not related to the already known effect for small local or wavelength scale boundary incisions discussed in Refs. [42-45] and promises higher $Q$ factors than cavities with a full slit in the cavity [46].

\section{B. Improving the $Q$ factor and suppression of unwanted modes}

The next goal is to selectively increase the $Q$ factors of only those modes supported by a transition through the notch. Therefore, a deformation is applied to the notched cavity
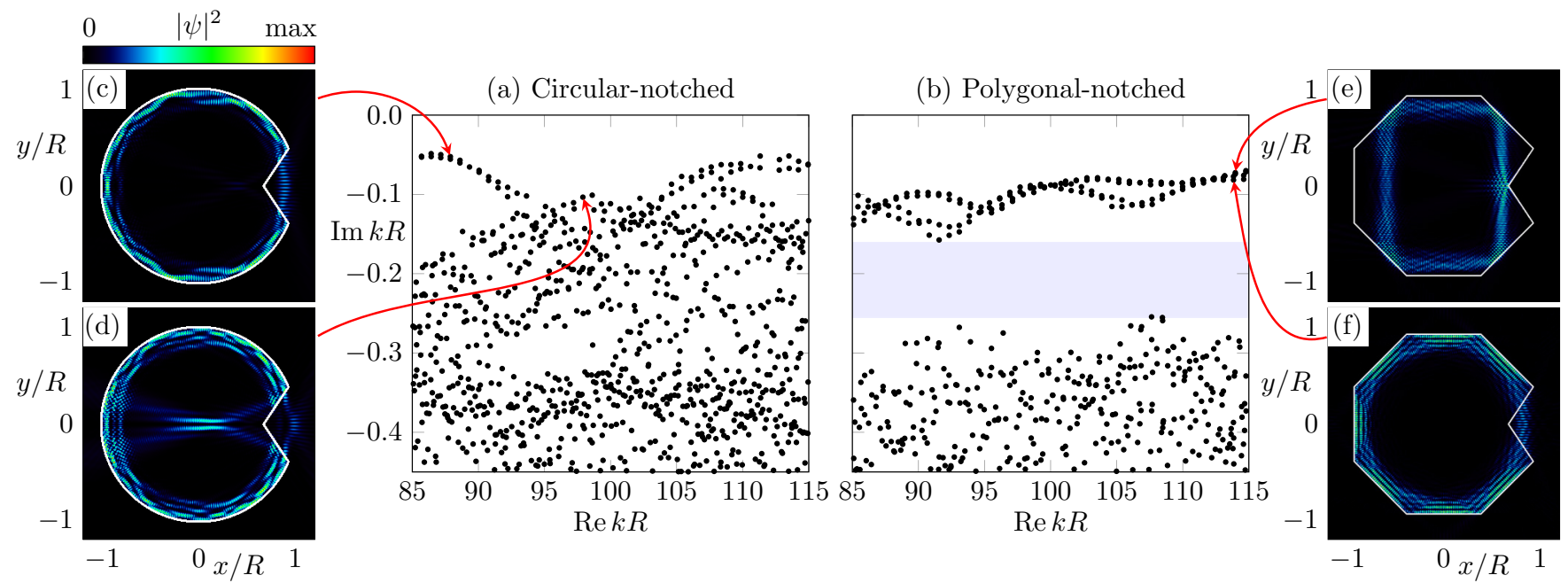

FIG. 2. Modes in the complex frequency plane of the (a) circular-notched and (b) polygonal-notched cavity. For several selected modes, the intensity pattern $|\psi|^{2}$ is shown in (c)-(f). 


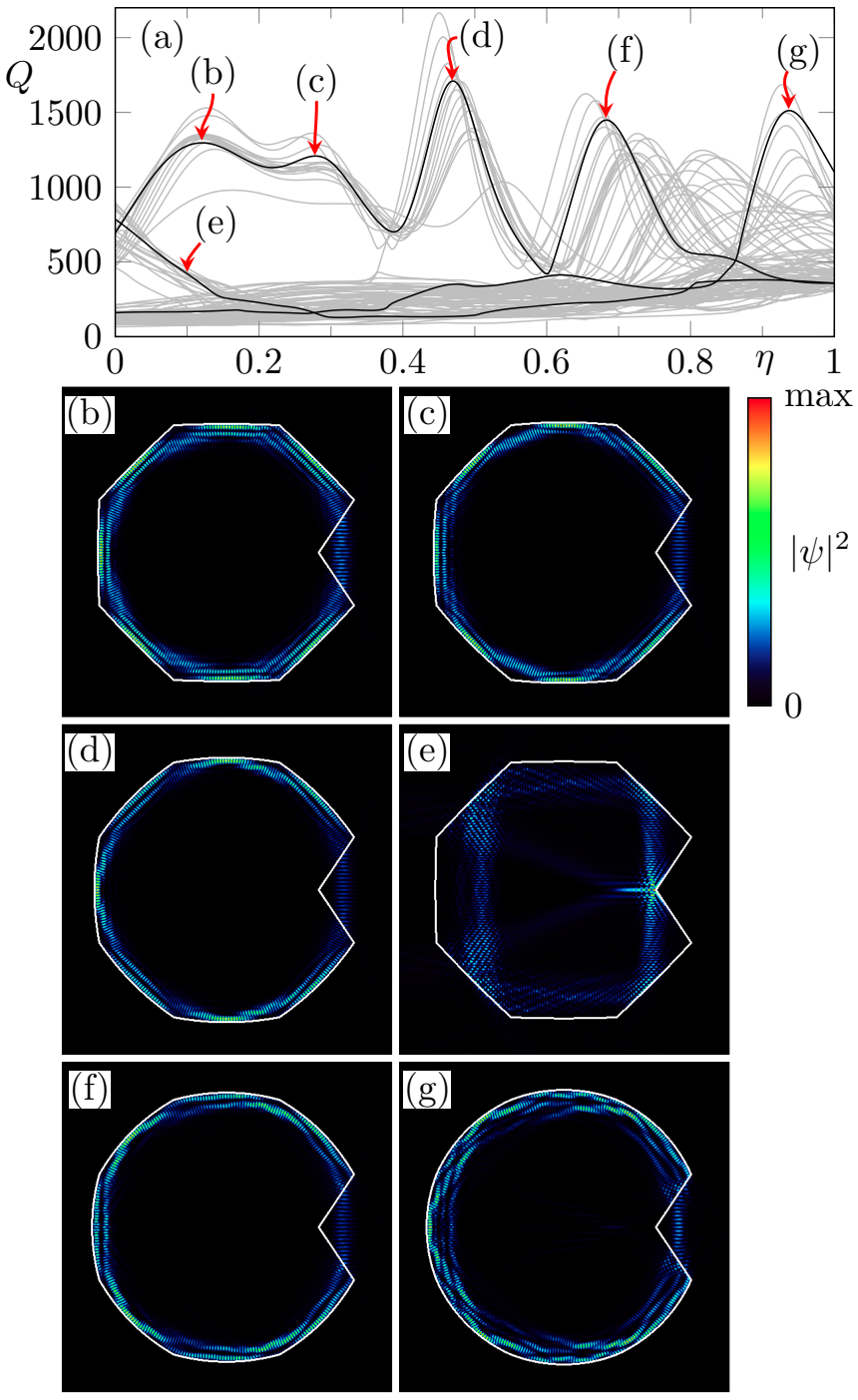

FIG. 3. For several modes with $\operatorname{Re} k R \in$ [85, 115], the $Q$-factor evolution with the deformation parameter $\eta$ [see Eq. (3)] is shown as gray and black curves. The (b)-(g) intensity mode patterns for the selected modes along the black curves in (a).

which smoothly interpolates from the polygonal-notched cavity to the circular-notched cavity by a parameter $\eta \in[0,1]$. More precisely, the radius function of the deformed cavity is constructed by

$$
r(\phi)=\eta r_{\text {circular-notched }}(\phi)+(1-\eta) r_{\text {polygonal-notched }}(\phi) .
$$

Note that via this deformation the notch itself is not changed. As shown in Fig. 3(a), starting from a mode in the polygonalnotched cavity [see Fig. 2(f)] the $Q$ factor for specific modes supported by the Brewster notch is improved. At the same time, the long-lived modes corresponding to the period-4 orbit [see Fig. 2(e)] experience a suppression in their $Q$ factors. For several values of the deformation parameter $\eta$, the $Q$ factor reaches a local maximum. As can be seen in Fig. 3, the corresponding modes localize along the periodic orbit passing the notch. Note that for a wide range of the deformation parameter $\eta \lesssim 0.6$, the long-lived modes are separated in the complex frequency plane from the short-lived modes.
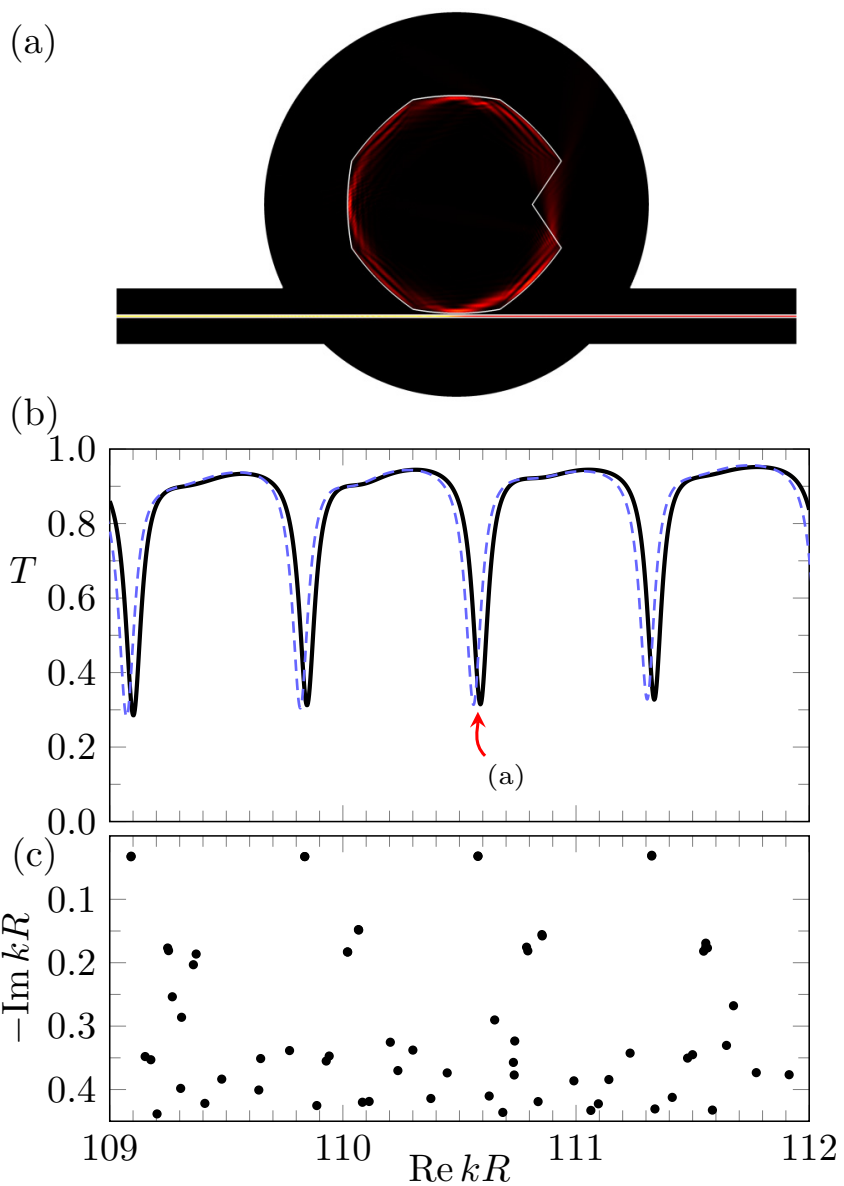

FIG. 4. Transmission spectrum of the deformed notched microdisk with a deformation parameter $\eta=0.468348$. For an excitation at the left edge of the waveguide, the square modulus of the wave function is shown in (a). The black background is the simulated domain. The solid black curve in (b) is the result from the COMSOL calculation for the transmission spectrum and the dashed curve is calculated using Meep. In (c), black dots are the BEM results for the deformed cavity without attached waveguide.

\section{Waveguide excitation}

In this section, we discuss the capability of the modes in the notched microdisk to be excited with an attached waveguide. To excite high- $Q$ modes, the deformation parameter $\eta$ is set to $\eta=0.468348$ corresponding to the central peak of the $Q$ factor in Fig. 3(a). Next to the notched cavity, a waveguide of width $h_{\mathrm{WG}}=0.02 R$ and refractive index $n_{\mathrm{WG}}=1.75$ is placed at a distance $d=0.015 R$. The transmission spectrum from the left to the right end of the waveguide is calculated with COMSOL MulTiPHYSiCs [47] and the results are shown as a solid curve in Fig. 4(b). The excitation pattern for the central peak in the spectrum is shown in Fig. 4(a). In addition, Fig. 4(c) shows the modes of the deformed notched cavity without a waveguide in the complex frequency plane. As each dip in the transmission spectrum corresponds to a long-lived mode of the notched cavity, it can be concluded that a waveguide excitation of the modes is feasible.

To support this claim further, we also performed finitedifference time-domain simulations with MEEP [48]. First, an 


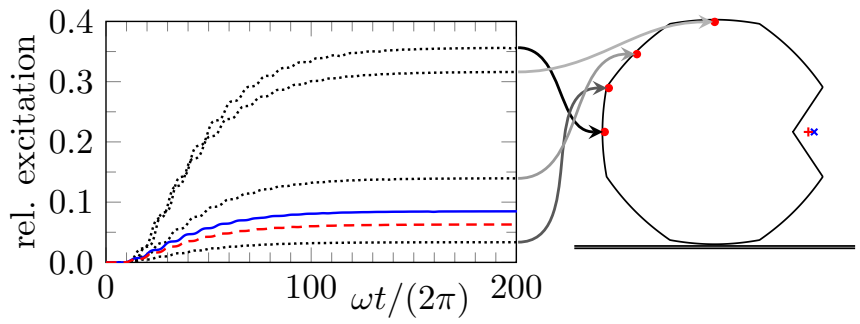

FIG. 5. Black dotted curves represent the evolution of the field intensities $|\psi|^{2}$ over time at several positions inside the cavity (see dots/arrows at the right panel). The blue solid (red dashed) curve show the field intensity in the notch at $x=0.85 R(x=0.8 R)$ and $y=$ 0 marked with a blue cross (red plus). The intensities are smoothed over the fast oscillations of the fields. The curves are normalized by the intensity excited in the center of the waveguide.

excitation at the left end of the waveguide with a Gaussian pulse in time is simulated to back up the previous calculations (in the frequency domain) of the transmission spectrum. As shown by the dashed curve in Fig. 4(b), generally a nice agreement to the COMSOL simulations is achieved and only a slight shift of the transmission spectrum due to the finite discretization is observed. In addition, the simulations with MEEP can show the time-resolved accumulation of the field intensity in the notch and in the cavity. Therefore, a waveguide excitation with a continuous source is used which is gradually turned on at $t=0$. The frequency of the source is $\omega R / c=110.528$ in correspondence to the central peak in Fig. 4(b). As shown in Fig. 5, the accumulated intensity strongly depends on the position in the cavity and nicely corresponds to the excitation pattern shown in Fig. 4(a). Especially, a considerable fraction of intensity inside the notch is excited. In addition, via the MEEP simulations, it is revealed that the process of intensity accumulation inside the cavity and inside the notch happen on the same timescale. For sensing applications, this ensures that detection via the notch has no temporal disadvantage, e.g., in terms of a delay, in comparison to the traditional sensing using the evanescent fields at the cavity's boundary.

\section{SENSING WITH THE NOTCHED CAVITY}

In this section, we verify the sensing capability of the notched cavity via the detection of a test particle in the vicinity of the cavity. Such a test particle, in general, causes a complex frequency splitting $\Delta k R=\left(k_{1}-k_{2}\right) R$ between two (almost) degenerate modes which refers as the signal. In particular, for the deformed notched cavity with $\eta=0.468348$, the two modes of even and odd parities have the almost degenerate frequencies $k_{1} R=110.579197-0.032334 i$ and $k_{2} R=110.579420-0.031619 i$. Note that such a finite initial splitting is the generic case for deformed (or fabricated) cavities [10]. If a test particle with refractive index $n_{\mathrm{TP}}=1.5$ and radius $r_{\mathrm{TP}}=0.005 R$ is moved in the notch along the $x$ axis, the caused frequency splitting is shown in Fig. 6(a). As expected, the splitting is large for a test particle placed in the middle of the notch where the modes have a considerable fraction of their intensities. An interesting observation is that the $Q$ factor can be increased slightly by the test particle if it is placed close to the periodic orbit, see Fig. 6(a). This effect,
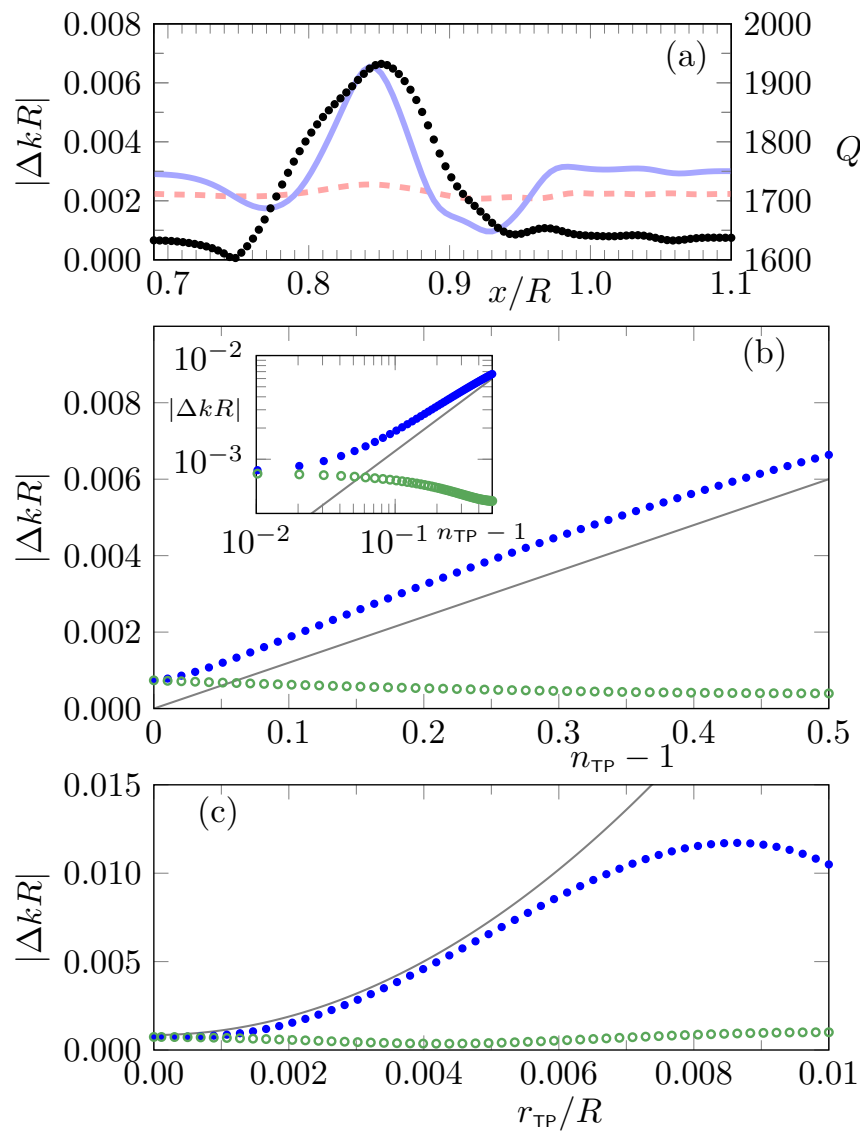

FIG. 6. In (a), the $Q$ factor of the even (odd) parity mode is shown as blue solid (red dashed) curve versus the test particle's position along the $x$ axis. Their corresponding frequency splitting modulus $|\Delta k R|$ is shown by black dots. The test particle's refractive index (radius) is $n_{\mathrm{TP}}=1.5\left(r_{\mathrm{TP}}=0.005 R\right)$. (b) The frequency splitting modulus $|\Delta k R|$ versus the test particle's refractive index $n_{\mathrm{TP}}$. The particle's position along the $x$ axis is at (blue dots) $x=0.85 R$ in the notch and (green empty circles) at a space of $d=0.002 R$ left to the cavity in the evanescent part of the field. The inset shows the same data but on a log-log scale. The same symbols show the frequency splitting modulus $|\Delta k R|$ versus the test particle's radius in (c) for fixed $n_{\mathrm{TP}}=1.5$. The gray solid curves are a guide to the eye and represent a linear [quadratic] behavior in (b) [(c)]. The deformation parameter of the notched cavity is $\eta=0.468348$.

however, strongly depends on the test particle's radius $r_{\mathrm{TP}}$ (not shown); as for larger $r_{\mathrm{TP}}$, modes inside the test particle itself lead to a mode coupling effect that typically spoils the $Q$ actor.

To benchmark the sensing performance, the test particle is placed at $(x, y)=(0.85 R, 0)$ and its refractive index is changed between $n_{\mathrm{TP}}=1$ (effectively no test particle) to $n_{\mathrm{TP}}=1.5$. Note that in this case, the test particle has a distance $d>0.146 R$ to the cavity's boundary. As shown in Fig. 6(b), the splitting depends roughly linearly on the refractive index and saturates if $n_{\mathrm{TP}}$ is close to unity since there is already a small initial splitting in the absence of the test particle. For comparison, in a traditional sensor based on whispering-gallery modes, the test particle only interacts with the evanescent leaking field of the mode. This scenario 
is captured by placing the test particle at the negative $x$ axis with a very narrow gap of $d=0.002 R$ to the left side of the cavity. The resulting change in the splitting and therefore the sensitivity in this case is much weaker, as shown in Fig. 6(b). For the evanescently coupled test particle, a partial reduction of the frequency splitting is observed. This is a known effect which can occur for specific test-particle realizations [49]. However, if the test particle's refractive index is increased further, this reduction in the splitting reverses such that for $n_{\mathrm{TP}} \approx 2.4$ it recurs to its initial value and increases further from thereon [not shown in Fig. 6(b)]. An interpretation of these curves within a two-mode model is given in Appendix A 1.

In Fig. 6(c), the dependency of the frequency splitting from the test particle's radius is shown for fixed $n_{\mathrm{TP}}=1.5$. Again, for the evanescently coupled test particle, the frequency splitting changes only marginally whereas the particle placed in the notch results in a generally larger splitting. For small $r_{\mathrm{TP}}$, this splitting roughly scales with $r_{\text {TP }}^{2}$ which is consistent with previous works on cavities at a (diabolic) degeneracy (see Supplemental Material of Refs. [50,51]).

\section{EXCEPTIONAL POINT BASED SENSING}

A possibility to improve the sensitivity of a microcavity sensor further is to take advantage of EPs in parameter space. Around the EP, the splitting between the involved modes increases with a characteristic root topology. Thus for a small perturbation, e.g., a small test particle with a refractive index close to unity, the splitting ensuing from the EP is expected to be larger than at an ordinary (diabolic) degeneracy. However, to make use of the EP-enhanced sensing, first, the system needs to be tuned to or very close to an EP. In several works, it has been shown that boundary deformations are a capable method to achieve an EP in whispering-gallery cavities [52-54]. In the same spirit, here, a boundary deformation is applied which breaks the mirror reflection symmetry of the cavity and therefore leads to a coupling between the modes of even and odd parities. More precisely, for the circular-notched cavity, a deformation according to the Fourier-transformed spiral [55] is used for angles $\phi \in[\pi / \nu, 2 \pi-\pi / \nu]$ where $\nu$ is again the number of reflections for the periodic orbit from which the notched cavity is constructed. The radius of the deformed cavity can be written in polar coordinates as

$$
\frac{r(\phi)}{R}=1-\epsilon \sum_{j=1}^{N_{\text {terms }}} \frac{(-1)^{j+1}}{j} \sin (j \nu \phi),
$$

where $\epsilon$ is the parameter for the deformation strength whereas the number of terms is fixed to $N_{\text {terms }}=4$. The notch itself remains unchanged. In general, a second parameter is needed to achieve an EP. For that purpose, a slight variation of the refractive index given by Eq. (2) is considered. In Fig. 7, it is shown that a variation of these two parameters around $n \approx 1.496516$ and $\epsilon \approx 0.005910$ reveals the characteristic square-root topology of an EP with two coalescing modes. It is mentioned that the system is not precisely at an EP for these parameters since two very close frequencies $k_{1} R \approx 90.07250-0.05343 i$ and $k_{2} R \approx 90.07163-0.05280 i$ can still be distinguished, see the red pluses in Fig. 7. However, both modes already
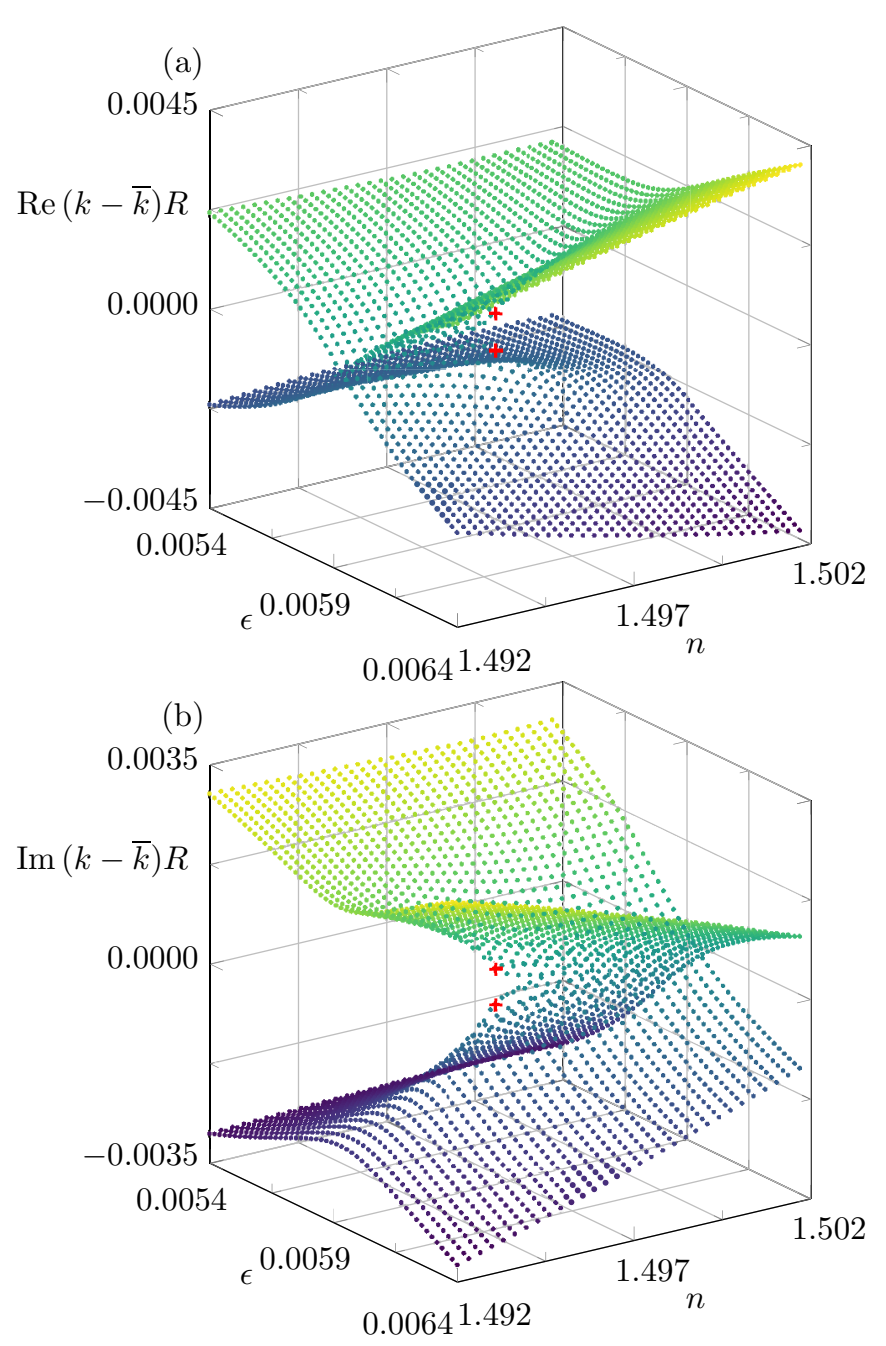

FIG. 7. The (a) real and (b) imaginary parts of the (shifted) complex frequencies of two modes in the asymmetrically deformed circular-notched cavity close to an EP. The cavity's refractive index $n$ and the deformation parameter $\epsilon$ are varied. Note that the frequencies are shown relative to the mean value $\bar{k} R=\left(k_{1}+k_{2}\right) R / 2$. The two complex frequencies with the smallest splitting are marked by red pluses.

show the relevant features for an EP including indistinguishable intensity patterns as shown in Figs. 8(a) and 8(b) and a characteristic chirality. The latter can be seen by calculating the current (Poynting vector)

$$
\vec{j} \propto \operatorname{Im}\left(\psi^{*} \nabla \psi\right)
$$

and from that the local angular momentum distribution via

$$
L_{z}=x j_{y}-y j_{x} .
$$

As shown in Figs. 8(c) and 8(d), the modes are copropagating in a clockwise (CW) direction. This finding is in good agreement to previous studies on whispering-gallery cavities where the abstract chirality around an EP [56] manifests in a physical chirality of the fields $[32,33,53,54]$.

Finally, this EP in the notched cavity can be utilized to enhance the sensing performance even though the cavity is not precisely at the EP. For a verification, again the test particle 


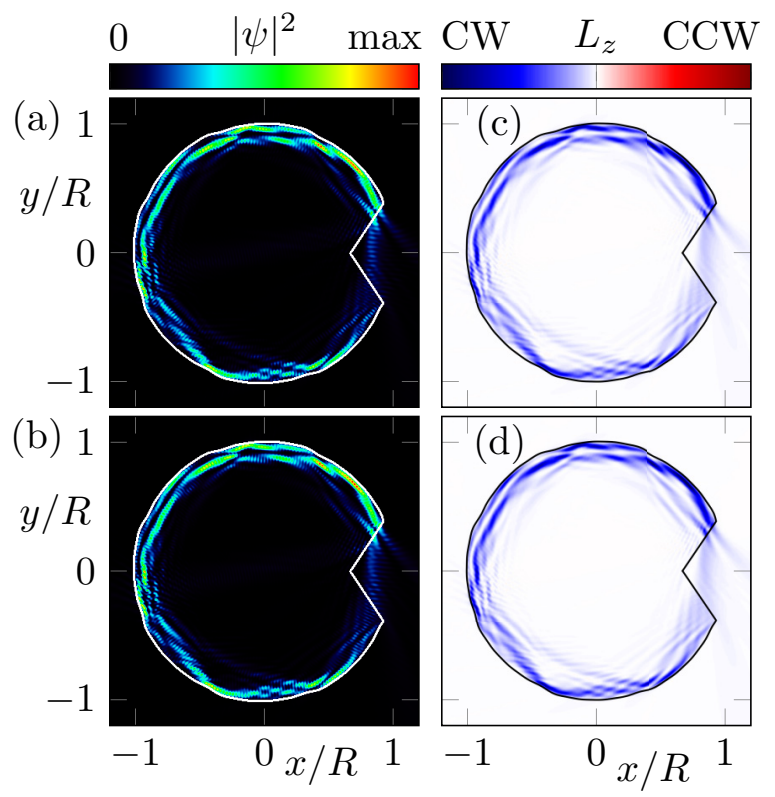

FIG. 8. (a), (b) Mode intensity pattern and (c), (d) angular momentum distribution (CW for clockwise and CCW for counterclockwise propagation) for the deformed-notched cavity at the EP with $n \approx 1.496516$ and $\epsilon \approx 0.005910$. The complex frequencies are (a), (c) $k_{1} R \approx 90.07250-0.05343 i$ and (b), (d) $k_{2} R \approx 90.07163-$ $0.05280 i$

with $r_{\mathrm{TP}}=0.005 R$ is placed at $x=0.85 R$ inside the notch and its refractive index $n_{\mathrm{TP}}$ is varied. As a result, the complex frequency splitting originated from the particle follows a square-root behavior as shown by the blue dots in Fig. 9 . Hence, a relatively strong splitting is already observed for test particles with a refractive index close to unity. Note that the exact values for the splitting in Fig. 9 cannot be directly compared to the values in Fig. 6(b) as the involved modes have a different real part of $k R$.

Alternatively, the test particle is placed at the left side of the cavity, where it can only interact with the evanescent leaking

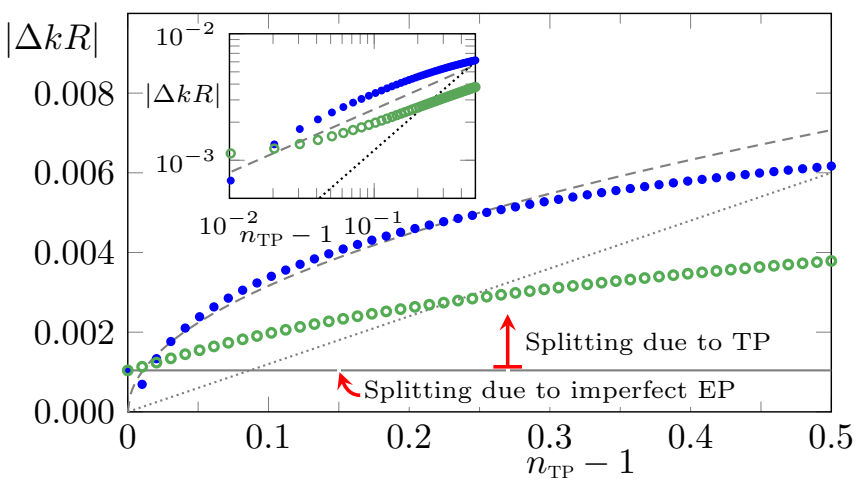

FIG. 9. The frequency splitting $|\Delta k R|$ is shown as a function of the test particle's (TP's) refractive index $n_{\mathrm{TP}}$ for the asymmetric notched cavity very close to the EP $(n \approx 1.496516$ and $\epsilon \approx$ $0.005910)$. The blue dots (green empty circles) are the results for a test particle at $x=0.85 R(d=0.002 R$ left to the cavity). The inset shows the same data on a log-log scale. A dashed (dotted) curve indicates the square root (linear) behavior as a guide to the eye. fields. In this case, the result is twofold. On the one hand, the splitting is still enhanced in comparison to a linear behavior due to the presence of an EP. This can be seen best in the inset of Fig. 9, which clearly indicates the square-root dependency. On the other hand, the overall splitting induced by the test particle is smaller, which again displays the superior sensitivity via the notch in comparison to the sensing with the evanescent fields.

In Appendix A 2, we also discuss the behavior of the frequency splitting for a cavity close to an EP within a two-mode model.

\section{SUMMARY}

In this paper, we introduced a cavity with a properly designed notch that combines two concepts for the confinement of light: total internal reflection and perfect transmission at Brewster's angle. Consequently, long-lived optical modes exist that are excitable via an attached waveguide and that have a considerable fraction of intensity in the free space of the notch. Via a boundary deformation, the $Q$ factor of the modes can be increased further. We also designed a fully asymmetric deformed notched cavity that exhibits an EP. This setup promises new advantages in the sensing performance of microcavities as it combines the sensitivity enhancement from EPs with the strong light-matter interaction of the modes inside the notch.

\section{ACKNOWLEDGMENT}

The authors acknowledge fruitful discussions with X. Jiang and L. Yang.

\section{APPENDIX A: TWO-MODE MODEL FOR THE FREQUENCY SPLITTING INDUCED BY A TEST PARTICLE}

In this Appendix, we discuss the behavior of the frequency splitting induced by a test particle within the scope of a two-mode approximation. Therefore, we reduce the mode equation (1) to an eigenvalue problem of a $2 \times 2$ matrix (Hamiltonian),

$$
H=H_{\text {cavity }}+H_{\mathrm{TP}},
$$

where $H_{\text {cavity }}\left(H_{\mathrm{TP}}\right)$ describe the notched cavity (test particle).

\section{Microcavity with a mirror reflection symmetry}

First, we focus on a cavity with a mirror reflection symmetry as in Sec. III. Therefore, the Hamiltonian is written in a standing wave basis of the two modes with even and odd parities. In the model, we assume the modes have a small initial splitting $2 \delta \in \mathbb{C}$ in their complex frequencies $\omega \pm \delta$ due to the noncircular shape of the cavity. As the test particle is placed along the axis $y=0$, it preserves the mirror-reflection symmetry and does not couple the two parity classes. In addition, we assume the particle being sufficiently small such that its impact on the mode with odd parity, that has node line along $y=0$, can be neglected. Note that this assumption is well supported by Fig. 6(a) where it is shown that the $Q$ factor of the odd-parity mode is almost independent from the test particle's position in the notch. Hence, we can write the 


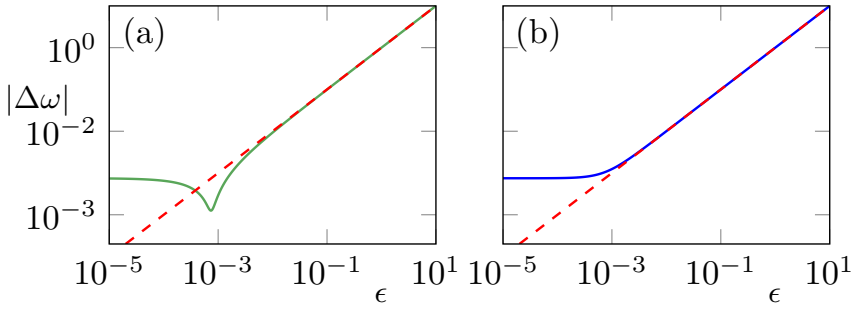

FIG. 10. Frequency splitting for the two-mode model close to a diabolic degeneracy for two different realizations [see Eq. (A2)] with (a) $\phi=1.35 \pi$ and (b) $\phi=1.9 \pi$ is shown as solid curves in a $\log -\log$ plot. The red dashed curves show the behavior of the corresponding diabolic degeneracy $(\delta=0)$.

Hamiltonian for the cavity and the test particle as [32]

$$
H_{\text {cavity }}=\left(\begin{array}{cc}
\omega+\delta & 0 \\
0 & \omega-\delta
\end{array}\right), \quad H_{\mathrm{TP}}=\epsilon\left(\begin{array}{cc}
0 & 0 \\
0 & e^{i \phi}
\end{array}\right),
$$

where $\epsilon>0$ determines the perturbation strength and $\phi \epsilon$ $[\pi, 2 \pi]$ describes the frequency shift and the induced losses. Both quantities, in general, depend on the test particle's position, its refractive index, its radius, and also on the mode's intensity at the particle's position. Hence, the frequency splitting due to the test particle is

$$
\Delta \omega=2 \delta-\epsilon e^{i \phi},
$$

which leads to

$$
|\Delta \omega|=\sqrt{4|\delta|^{2}-4 \epsilon \operatorname{Re}\left(\delta e^{-i \phi}\right)+\epsilon^{2}} .
$$

In particular, for $|\delta| \ll \epsilon$ it follows that

$$
|\Delta \omega|=\epsilon
$$

as the leading order term. For simplicity, we select randomly chosen values $\phi=1.35 \pi$ and $\phi=1.9 \pi$ for $10^{4} \delta \approx-1.1-$ $3.6 i$ in Fig. 10 to discuss possible scenarios. First, for both realizations, the splitting shows the expected linear scaling for $\epsilon \gg|\delta|$ and saturates to the initial splitting for $\epsilon \ll|\delta|$. However, in the intermediate regime the behavior is different, i.e., in Fig. 10(a) a partial cancellation of the splitting occurs while the transition to a linear splitting in Fig. 10(b) is a monotone function of the perturbation strength. Both scenarios can now be identified in Fig. 6(b), where the perturbation strength is encoded in the refractive index of the test particle. However, it needs to be kept in mind that an evanescently coupled test particle is a much weaker perturbation to the system than a test particle inside the notch where the mode's intensity is high, i.e., $\epsilon_{\text {evanescent }} \ll \epsilon_{\text {notch }}$ for the same $n_{\text {TP }}$. Therefore, the refractive index variation $n_{\mathrm{TP}} \in[1,1.5]$ for the evanescently coupled test particle can only cover the regime of very small perturbation strengths $\epsilon$, where the reduction of the splitting is observed.

\section{Microcavity at an exceptional point}

In this part of the Appendix, we discuss the frequency splitting behavior in a cavity with asymmetric backscattering between $\mathrm{CW}$ and counter-clockwise $(\mathrm{CCW})$ propagating modes as observed in Sec. IV. The cavity is close to an EP so its Hamiltonian in the traveling wave basis $[(1,0)$ for $\mathrm{CCW}$
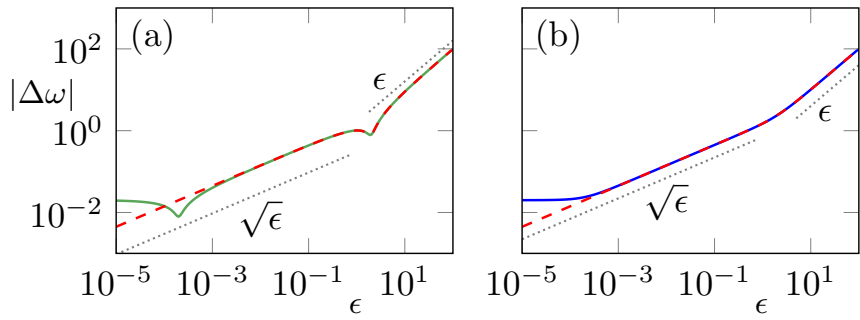

FIG. 11. The solid curves show the frequency splitting for the two-mode model close to an EP for two different realization of $H_{\mathrm{TP}}$ in (a) and (b), respectively, in a log-log plot. The dashed curves illustrate the frequency splitting exactly at the EP with $b=0$. The dotted lines serve as a guide to the eye and indicate a linear and square-root behavior.

propagation and $(0,1)$ for $\mathrm{CW}$ propagation] can be written with $a, b, \omega \in \mathbb{C}$ as [32]

$$
H_{\text {cavity }}=\left(\begin{array}{ll}
\omega & a \\
b & \omega
\end{array}\right),
$$

where $|b| \ll|a|$ indicates that scattering from $\mathrm{CW}$ to $\mathrm{CCW}$ is preferred over the reversed process. For $b=0$ and $a \neq 0$, the system would be exactly at the EP. However, we assume a finite but small $b$ such that the modes in the cavity without test particles have the initial frequencies $\omega \pm \sqrt{a b}$. The Hamiltonian for the coupling to the particle can be written in the traveling-wave basis as [32]

$$
H_{\mathrm{TP}}=\left(\begin{array}{cc}
\alpha & \alpha \\
\alpha & \alpha
\end{array}\right)
$$

with $\alpha=\epsilon \exp (i \phi) / 2$ which leads to a splitting

$$
\Delta \omega=2 \sqrt{(a+\alpha)(b+\alpha)} .
$$

For simplicity, we again select two sets of random numbers for these matrices to illustrate characteristic behaviors of the frequency splitting in Fig. 11. Here, three domains can be identified: (i) For extremely small $\epsilon$, the splitting is dominated by a saturation to the initial splitting. Therefore, in a $\log$-log plot, clear differences to the behavior associated with an (ideal) EP are present. (ii) A regime where the splitting scales with a square root. This can be seen by approximating Eq. (A8) for small $|b|, \epsilon \ll|a|$, which yields

$$
\Delta \omega \approx 2 \sqrt{a\left(b+\epsilon e^{i \phi} / 2\right)},
$$

from which we can identify the leading term for the splitting modulus as

$$
|\Delta \omega|=\sqrt{2|a|} \sqrt{\epsilon} .
$$

For applications, this is the interesting regime as the beneficial square-root scaling can be utilized even though the EP is not achieved exactly. This is also the regime mainly shown in Fig. 9. (iii) The regime of large perturbations where linear terms lead to a scaling comparable to a diabolic point. For applications, this is again the uninteresting regime where EP enhanced sensing is not present (and not necessary). In general, for such large perturbations, effects beyond a two-mode model are likely, e.g., (avoided) crossings with modes inside the test particle or other modes within the notched cavity. 
[1] R. K. Chang and A. J. Campillo, Optical Processes in Microcavities, Advanced Series in Applied Physics, Vol. 3 (World Scientific, Singapore, 1996).

[2] K. J. Vahala, Optical Microcavities, Advanced Series in Applied Physics, Vol. 5 (World Scientific, Singapore, 2004).

[3] A. M. Armani, R. P. Kulkarni, S. E. Fraser, R. C. Flagan, and K. J. Vahala, Label-free, single-molecule detection with optical microcavities, Science 317, 783 (2007).

[4] F. Vollmer and S. Arnold, Whispering-gallery-mode biosensing: label-free detection down to single molecules, Nat. Methods 5, 591 (2008).

[5] F. Vollmer, S. Arnold, and D. Keng, Single virus detection from the reactive shift of a whispering-gallery mode, Proc. Nat. Acad. Sci. USA 105, 20701 (2008).

[6] F. Vollmer and L. Yang, Label-free detection with high- $Q$ microcavities: A review of biosensing mechanisms for integrated devices, Nanophotonics 1, 267 (2012).

[7] G. Guan, S. Arnold, and M. V. Otugen, Temperature measurements using a microoptical sensor based on whispering gallery modes, AIAA J. 44, 2385 (2006).

[8] Sung Hyun Nam and Shizhuo Yin, High-temperature sensing using whispering gallery mode resonance in bent optical fibers, IEEE Photon. Technol. Lett. 17, 2391 (2005).

[9] L. Xu, X. Jiang, G. Zhao, D. Ma, H. Tao, Z. Liu, F. G. Omenetto, and L. Yang, High-Q silk fibroin whispering gallery microresonator, Opt. Express 24, 20825 (2016).

[10] S. Sunada and T. Harayama, Design of resonant microcavities: Application to optical gyroscopes, Opt. Express 15, 16245 (2007).

[11] J. U. Nöckel, A. D. Stone, G. Chen, H. L. Grossman, and R. K. Chang, Directional emission from asymmetric resonant cavities, Opt. Lett. 21, 1609 (1996).

[12] J. U. Nöckel and A. D. Stone, Ray and wave chaos in asymmetric resonant optical cavities, Nature (London) 385, 45 (1997).

[13] J. Wiersig and M. Hentschel, Combining Directional Light Output and Ultralow Loss in Deformed Microdisks, Phys. Rev. Lett. 100, 033901 (2008).

[14] Q. J. Wang, C. Yan, N. Yu, J. Unterhinninghofen, J. Wiersig, C. Pflügl, L. Diehl, T. Edamura, M. Yamanishi, H. Kan, and F. Capasso, Whispering-gallery mode resonators for highly unidirectional laser action, Proc. Natl. Acad. Sci. USA 107, 22407 (2010).

[15] S. Shinohara, M. Hentschel, J. Wiersig, T. Sasaki, and T. Harayama, Ray-wave correspondence in limaçon-shaped semiconductor microcavities, Phys. Rev. A 80, 031801(R) (2009).

[16] F. Albert, C. Hopfmann, A. Eberspächer, F. Arnold, M. Emmerling, C. Schneider, S. Höfling, A. Forchel, M. Kamp, J. Wiersig, and S. Reitzenstein, Directional whispering gallery mode emission from Limaçon-shaped electrically pumped quantum dot micropillar lasers, Appl. Phys. Lett 101, 021116 (2012).

[17] Q. J. Wang, C. Yan, L. Diehl, M. Hentschel, J. Wiersig, N. Yu, C. Pflügl, M. A. Belkin, T. Edamura, M. Yamanishi, H. Kan, and F. Capasso, Deformed microcavity quantum cascade lasers with directional emission, New J. Phys. 11, 125018 (2009).

[18] M. Schermer, S. Bittner, G. Singh, C. Ulysee, M. Lebental, and J. Wiersig, Unidirectional light emission from low-index polymer microlasers, Appl. Phys. Lett. 106, 101107 (2015).

[19] X.-F. Jiang, Y.-F. Xiao, C.-L. Zou, L. He, C.-H. Dong, B.-B. Li, Y. Li, F.-W. Sun, L. Yang, and Q. Gong, Highly unidirectional emission and ultralow-threshold lasing from on-chip ultrahigh$Q$ microcavities, Adv. Mater. 24, OP260 (2012).

[20] T. J. Kippenberg, R. Holzwarth, and S. A. Diddams, Microresonator-based optical frequency combs, Science 332, 555 (2011).

[21] M.-G. Suh and K. Vahala, Gigahertz-repetition-rate soliton microcombs, Optica 5, 65 (2018).

[22] A. Rueda, F. Sedlmeir, M. Kumari, G. Leuchs, and H. G. L. Schwefel, Resonant electro-optic frequency comb, Nature $\mathbf{5 6 8}$, 378 (2018).

[23] P. Miao, Z. Zhang, J. Sun, W. Walasik, S. Longhi, N. M. Litchinitser, and L. Feng, Orbital angular momentum microlaser, Science 353, 464 (2016).

[24] H. Cao and J. Wiersig, Dielectric microcavities: Model systems for wave chaos and non-Hermitian physics, Rev. Mod. Phys. 87, 61 (2015).

[25] S. Bittner, S. Guazzotti, Y. Zeng, X. Hu, H. Yılmaz, K. Kim, S. S. Oh, Q. J. Wang, O. Hess, and H. Cao, Suppressing spatiotemporal lasing instabilities with wave-chaotic microcavities, Science 361, 1225 (2018).

[26] X. Jiang, L. Shao, S.-X. Zhang, X. Yi, J. Wiersig, L. Wang, Q. Gong, M. Lončar, L. Yang, and Y.-F. Xiao, Chaos-assisted broadband momentum transformation in optical microresonators, Science 358, 344 (2017).

[27] Y.-F. Xiao, C.-L. Zou, Q. Gong, and L. Yang, Ultra-high-Q Optical Microcavities (World Scientific, Singapore, 2020).

[28] J. Kullig, X. Jiang, L. Yang, and J. Wiersig, Microstar cavities: An alternative concept for the confinement of light, Phys. Rev. Res. 2, 012072(R) (2020).

[29] T. Kato, Perturbation Theory for Linear Operators (Springer, New York, 1966).

[30] C. Dembowski, H.-D. Gräf, H. L. Harney, A. Heine, W. D. Heiss, H. Rehfeld, and A. Richter, Experimental Observation of the Topological Structure of Exceptional Points, Phys. Rev. Lett. 86, 787 (2001).

[31] W. D. Heiss, The physics of exceptional points, J. Phys. A: Math. Theor. 45, 444016 (2012).

[32] J. Wiersig, Structure of whispering-gallery modes in optical microdisks perturbed by nanoparticles, Phys. Rev. A 84, 063828 (2011).

[33] B. Peng, Ş. K. Özdemir, M. Liertzer, W. Chen, J. Kramer, H. Y1lmaz, J. Wiersig, S. Rotter, and L. Yang, Chiral modes and directional lasing at exceptional points, Proc. Nat. Acad. Sci. USA 113, 6845 (2016).

[34] J. Wiersig, Enhancing the Sensitivity of Frequency and Energy Splitting Detection by Using Exceptional Points: Application to Microcavity Sensors for Single-Particle Detection, Phys. Rev. Lett. 112, 203901 (2014).

[35] J. Wiersig, Sensors operating at exceptional points: General theory, Phys. Rev. A 93, 033809 (2016).

[36] W. Chen, Ş. K. Özdemir, G. Zhao, J. Wiersig, and L. Yang, Exceptional points enhance sensing in an optical microcavity, Nature 548, 192 (2017).

[37] J. Wiersig, Review of exceptional point-based sensors, Photon. Res. 8, 1457 (2020).

[38] J. D. Jackson, Classical Electrodynamics (John Wiley and Sons, New York, 1962).

[39] J. Wiersig, Boundary element method for resonances in dielectric microcavities, J. Opt. A: Pure Appl. Opt. 5, 53 (2003). 
[40] A. Schlehahn, F. Albert, C. Schneider, S. Höfling, S. Reitzenstein, J. Wiersig, and M. Kamp, Mode selection in electrically driven quantum dot microring cavities, Opt. Express 21, 15951 (2013).

[41] S. Boriskina, T. Benson, P. Sewell, and A. Nosich, Highly efficient design of spectrally engineered whispering-gallerymode microlaser resonators, Opt. Quantum Electron. 35, 545 (2003).

[42] J. Wiersig, Perturbative approach to optical microdisks with a local boundary deformation, Phys. Rev. A 85, 063838 (2012).

[43] M. Fujita and T. Baba, Microgear lasers, Appl. Phys. Lett. 80, 2051 (2002).

[44] S. V. Boriskina, T. M. Benson, P. Sewell, and A. Nosich, $Q-$ factor and emission pattern control of the WG modes in notched microdisk resonators, IEEE J. Sel. Top. Quantum Electron. 12, 52 (2006).

[45] S. V. Boriskina, T. M. Benson, P. D. Sewell, and A. I. Nosich, Directional emission, increased free spectral range, and mode $Q$-factors in 2-D wavelength-scale optical microcavity structures, IEEE J. Sel. Top. Quantum Electron. 12, 1175 (2006).

[46] T. Siegle, M. Remmel, S. Krämmer, and H. Kalt, Split-disk micro-lasers: Tunable whispering gallery mode cavities, APL Photonics 2, 096103 (2017).

[47] COMSOL Multiphysics ${ }^{\circledR}$ v. 5.4. www.comsol.com, COMSOL AB, Stockholm, Sweden.

[48] A. F. Oskooi, D. Roundy, M. Ibanescu, P. Bermel, J. D. Joannopoulus, and S. G. Johnson, Meep: A flexible free- software package for electromagnetic simulations by the FDTD method, Comput. Phys. Commun. 181, 687 (2010).

[49] J. Zhu, Ş. K. Özdemir, L. He, and L. Yang, Controlled manipulation of mode splitting in an optical microcavity by two Rayleigh scatterers, Opt. Express 18, 23535 (2010).

[50] Q. Zhong, J. Ren, M. Khajavikhan, D. N. Christodoulides, Ş. K. Özdemir, and R. El-Ganainy, Sensing with Exceptional Surfaces in Order to Combine Sensitivity with Robustness, Phys. Rev. Lett. 122, 153902 (2019).

[51] Q. Zhong, J. Kou, Ş. K. Özdemir, and R. El-Ganainy, Hierarchical Construction of Higher-Order Exceptional Points, Phys. Rev. Lett. 125, 203602 (2020).

[52] S.-B. Lee, J. Yang, S. Moon, S.-Y. Lee, J.-B. Shim, S. W. Kim, J.-H. Lee, and K. An, Observation of an Exceptional Point in a Chaotic Optical Microcavity, Phys. Rev. Lett. 103, 134101 (2009).

[53] C.-H. Yi, J. Kullig, and J. Wiersig, Pair of Exceptional Points in a Microdisk Cavity Under an Extremely Weak Deformation, Phys. Rev. Lett. 120, 093902 (2018).

[54] J. Kullig and J. Wiersig, High-order exceptional points of counterpropagating waves in weakly deformed microdisk cavities, Phys. Rev. A 100, 043837 (2019).

[55] J. Kullig and J. Wiersig, Frobenius-Perron eigenstates in deformed microdisk cavities: non-Hermitian physics and asymmetric backscattering in ray dynamics, New J. Phys. 18, 015005 (2016).

[56] W. D. Heiss and H. L. Harney, The chirality of exceptional points, Eur. Phys. J. D 17, 149 (2001). 